\title{
Full-Face-Behandlung für natürliche Ergebnisse
}

In der ästhetischen Dermatologie verliert die alleinige Korrektur einzelner Falten immer mehr an Bedeutung. Der Trend geht heute zu einer ganzheitlichen Verjüngung der Gesichtszüge, bei der eine Volumenaugmentation im Mittelpunkt steht.

Alterungsprozesse im Gesicht gehen mit Veränderungen der Fettkomparti-

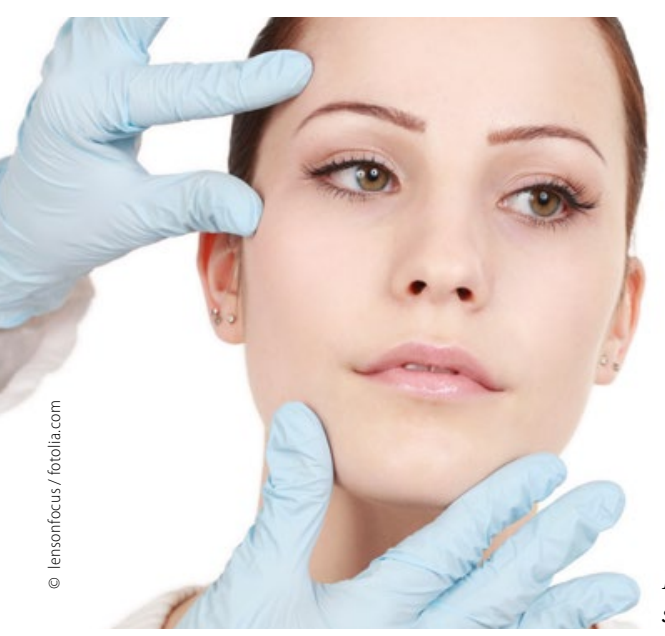

Die ganzheitliche Verjüngung der Gesichtszüge schafft natürliche Behandlungsergebnisse.

mente einher, erklärte PD Dr. Gerd G. Gauglitz, Leiter der Abteilung Ästhetische Dermatologie und Lasermedizin, Ludwig-Maximilians-Universität München. Besonders im oberen Mittelgesicht kommt es zu einer Atrophie der tieferen Fettdepots. Zudem sinken oberflächlichen Depots ab und kumulieren beispielsweise an der Nasolabialfalte. Darüber hinaus verändern sich knöcherne Strukturen, beispielsweise an der Augenhöhle. Um solchen Veränderungen entgegenzuwirken, setzt sich heute zunehmend eine Full-Face-Behandlung durch, so Gauglitz.

Für die Volumenaugmentation steht eine Vielzahl an Füllmaterialien zur Verfügung. Gauglitz empfahl, Produkte zu wählen, die in klinischen Studien gut untersucht sind. Dazu zählen z. B. Belotero ${ }^{\circ}$ Volume mit Hyaluronsäure sowie Radiesse mit dem Wirkstoff Calciumhydroxylapa-

Peel-Akademie

\section{Hyperpigmentierungen und Hautbarrierestörungen}

Die Dermasence Peel-Akademie bietet im 2. Halbjahr aufbauend auf der Dermasence Peel-Basis-Schulung zwei neue Seminare an: Das Seminar „Hyperpigmentierungen - ein lösbares Problem“ beschäftigt sich mit der differenzierten Betrachtung von Hyperpigmentierungen und deren Ursachen. Gerade nach Operationen, bei entzündlicher Akne, hormonellen Veränderungen oder starker Lichteinstrahlung kann es zu flächigen Pigmentveränderungen der Haut kommen.

In dem Seminar „Fruchtsäure-Peels bei Hautbarrierestörungen“ werden wichtige Störfaktoren für die Hautbarriere erläutert. Im besonderen Fokus stehen hier die Indikationen Akne, Atopie, Rosazea,
Xerosis und reife Haut. Dermasence Peelings und medizinische Hautpflege können therapiebegleitend zur Stabilisierung der Hautbarriere und Verbesserung der Hauterkrankung beitragen. In der LiveBehandlung werden praktische Tipps zur Vorgehensweise demonstriert.

Weitere Informationen zu den beiden Seminaren können unter der Telefonnummer 0251/91599-100 oder per E-Mail unter info@dermasence.de abgerufen werden. Es sollte eine rasche Anmeldung erfolgen die Teilnehmerzahl ist begrenzt.

\section{Dermasence Peel-Kompendium}

Dermasence hat sich mit engagierten Dermatologen zusammengeschlossen und das tit. Beide Produkte weisen eine sehr gute Verträglichkeit auf und sind flexibel einsetzbar, betonte Gauglitz.

Der Filler mit Calciumhydroxylapatit zeichnet sich durch eine gute Hebekapazität aus und ist zum Volumenlifting und zur Konturierung geeignet. Auch stark bewegte Areale können damit behandelt werden. Das Produkt führt zu einer nachhaltigen Kollagenstimulation.

Der Hyaluronfiller ist ebenfalls zum Ausgleich von Volumendefiziten einsetzbar. Das Produkt weist eine sehr gute Gewebeintegration auf. Der Filler ist leicht zu injizieren und sorgt dank seiner guten Modelliereigenschaften für weiche und runde Übergänge.

Dr. Welf Prager, niedergelassener Dermatologe in Hamburg, demonstrierte live die praktische Anwendung. Wichtig sind gute anatomische Kenntnisse des Behandlers. Die Verwendung stumpfer Kanülen kann dem Risiko für Gefäßverletzungen entgegenwirken. Durch die Kombination der beiden Filler-Produkte lassen sich besonders schöne, natürliche Ergebnisse erzielen. Angelika Bauer-Delto

Mittagsseminar "Full-face-Behandlungen - unser Weg zu natürlichen Ergebnissen“ anlässlich der 48. Tagung der Deutschen Dermatologischen Gesellschaft, Berlin, 1.5.2015; Veranstalter: Merz Pharmaceuticals

Peel-Kompendium ins Leben gerufen. Es sind Erfahrungen aus Sicht der Therapeuten aufgeführt, die von überzeugenden Fotodokumentationen unterstützt werden. Interessierte, die das kombinierte Peeling in der Praxis einsetzen, finden Anregungen für neue Herangehensweisen. Indikationen wie z. B. Akne comedonica, Akne juveniles, aktinische Keratosen, Keloide, Melasma/ Hyperpigmentierungen, Mykosen oder Onychogryposen füllen bereits einen Teil der Broschüre. Dermasence nimmt für die nächsten Ausführungen selbstverständlich noch weitere „Peel-Rezepte“ und neue Indikationen auf. Interessierte Peel-Anwender mit neuen Peel-Erfahrungen und sichtbaren Behandlungserfolgen können sich unter der angegebenen Kontaktadresse melden und ein Teil des Peel-Kompendiums werden. Um detaillierte Einblicke zu erhalten, können Anwender die Broschüre bei P\&M Cosmetics anfordern.

Nach Informationen von P\&M Cosmetics 